Post-print version of:

Publisher: Springer

Journal paper: International Journal on Interactive Design and Manufacturing, 10(2): 105-117

Title: Interactive design of dental implant placements through CAD-CAM technologies: from 3D imaging to additive manufacturing

Authors: S. Barone, M. Casinelli, M. Frascaria, A. Paoli, A. V. Razionale

Creative Commons Attribution Non-Commercial No Derivatives License

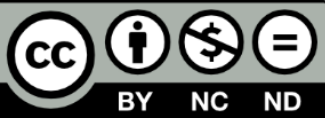

DOI Link: https://doi.org/10.1007/s12008-014-0229-0 


\title{
Interactive design of dental implant placements through CAD-CAM technologies: from 3D imaging to additive manufacturing
}

\author{
S.Barone, M. Casinelli, M. Frascaria, A. Paoli, A. V. Razionale
}

\begin{abstract}
In the field of oral rehabilitation, the combined use of 3D imaging technologies and computer-guided approaches allows the development of reliable tools to be used in preoperative assessment of implant placement. In particular, the accurate transfer of the virtual planning into the operative field through surgical guides represents the main challenge of modern dental implantology. Guided implant positioning allows surgical and prosthetic approaches with minimal trauma by reducing treatment time and decreasing patient's discomfort.

This paper aims at defining a CAD/CAM framework for the accurate planning of flapless dental implant surgery. The system embraces three major applications: 1) freeform modelling, including 3D tissue reconstruction and 2D/3D anatomy visualization, 2) computer-aided surgical planning and customised template modelling, 3) additive manufacturing of guided surgery template.

The tissue modelling approach is based on the integration of two maxillofacial imaging techniques: tomographic scanning and surface optical scanning. A 3D virtual maxillofacial model is created by matching radiographic data, captured by a CBCT scanner, and surface anatomical data, acquired by a structured light scanner. The pre-surgical planning process is carried out and controlled within the CAD application by referring to the integrated anatomical model. A surgical guide is then created by solid modelling and manufactured by additive techniques.

Two different clinical cases have been approached by inserting 11 different implants. CAD-based planned fixture placements have been transferred into the clinical field by customised surgical guides, made of a biocompatible resin and equipped with drilling sleeves.
\end{abstract}

Keywords: oral rehabilitation, computer-assisted dental implantology, freeform solid modelling, biomedical imaging, additive manufacturing. 
In the field of oral rehabilitation, the use of dental implants has progressively evolved replacing removable dentures in the treatment of edentulous patients. The traditional approach to dental implant surgery is based on raising flap procedures, which combine two-dimensional radiographs with visual inspection and clinical palpation. Current trends in implant dentistry are focused on the development of less invasive procedures with less post-operative bleeding, reduced surgical intervention time and greater healing rates [1,2]. In recent years, many clinicians have proposed various flapless approaches, which are generally based on using a tissue punch device to directly access the alveolar ridge [3] [4,5]. However, these techniques may cause serious injuries owing to the inability to visualize vital structures combined with difficulties in the evaluation of the alveolar bone contours. Therefore, an appropriate pre-surgical planning should always be performed by combining anatomical and prosthetic considerations in order to guarantee a successful placement and a predictable treatment outcome.

Recently, computer-guided methodologies have been developed with the aim at defining reliable tools for the virtual preoperative assessment of implant placement [1,6-11]. Information regarding anatomical constraints, bone volume and bone quality can be evaluated by processing three-dimensional data recorded through Computed Tomography (CT) or Cone Beam Computed Tomography (CBCT) techniques [12]. The maxillofacial digital data can be processed by interactive software to simulate the implant placement taking into account both anatomical and restorative demands. The virtual implant placements are then transferred to patients through customized computer-designed surgical templates. A guiding template consists of a customised resin-made model with implant system-related mounts for fixture installation, additional sleeves for fixation screw installation, drill keys of different inner diameters, and depth-calibrated drills to prepare the osteotomies. Moreover, surgical guides have to closely fit hard and soft tissues in a unique and stable position in order to accurately transfer the pre-operative treatment plan $[13,14]$.

Although several surgical guiding methodologies have been proposed in technical literature $[15,16]$, guided implant surgery is far away from being considered a standard routine. Deviation between planning and actual implant positions might occur at different treatment stages: tomographic scanning, transfer of planning data, manufacturing and positioning of the surgical guide, implants installing [17]. The overall accuracy is the sum of the errors encountered during the entire process: from anatomical data recording to customised template manufacturing. Throughout the implant treatment planning, the most critical issue consists in transferring CT information into surgical field. The sole use of CT data might not guarantee a proper fit between surgical templates and mucosa- (soft) and/or tooth- (hard) tissues [16,18,19], since tomographic data do not provide the oral tissues with accuracy as required for dental implant surgery [20]. The resulting geometrical deviations of implant positions between planning and intervention stages could cause alveolar bone dehiscence, fenestrations and/or irreversible damages of anatomical structures, such as sensory nerves.

In this paper, a CAD/CAM framework for accurate 3D planning of dental implant placements has been developed by integrating two different maxillofacial imaging modalities: CBCT scanning and surface optical scanning. The integration of volumetric radiographic anatomical data with $3 \mathrm{D}$ surface information allows 
accurate reconstruction of patient's maxillofacial shapes, which are used as references for the implant treatment planning. Usually, the volumetric reconstruction from CBCT data needs appropriate segmentation processes, whose results rely on the adopted mathematical algorithm, the spatial and contrast resolution of the image slices and the technical skills of the operator in selecting the optimal threshold value. Moreover, the presence of streak artifacts owing to metal restorations and/or orthodontic fixed appliances may significantly obscure dental arches in the tomographic scan. The proposed approach enables the optimization of the CBCT data segmentation process, since an optimal threshold value is automatically computed by minimizing the discrepancies between the digital models obtained by the two different scanning technologies [21].

The recording and modeling tools are integrated within a framework dedicated to 3D design of implant prosthetics taking into account aesthetic, biomechanical and functional demands. Specifically, the overall system applies computer-aided technologies including biomedical image processing, freeform tissue modelling, computer-aided design for surgical planning, and rapid manufacturing for guiding template production.

The proposed approach is based on an enhanced interaction between the clinician and the patient anatomy, though an extensive use of digital and physical models. In particular, the aim of paper relies on the definition of a CAD-CAM framework where the continuous interaction between expert's knowledge, clinical constraints, 3D anatomical virtual models, rapid prototyping techniques and optical surface measurements can support the decision-making process during the surgical guide design and manufacturing. Moreover, the methodology allows prosthetically-driven implant placements, which ensure precise fixtures' positioning and predictable prosthetic outcomes. This is assured by using an optical scanner as supporting tool to monitor the geometrical inaccuracies throughout the whole planning process. The use of CAD/CAM techniques for manufacturing surgical drill guides assists the physical transfer of the implant planning to the patient's mouth, thus improving accuracy and feasibility of the final implant placement. In this paper, the methodology has been experimented in two different clinical contexts. The medical results have confirmed the validity of the proposed approach.

\section{$2 \quad$ Materials and methods}

The design of an implant placement should take into account anatomical, functional and aesthetical requirements. This paper aims at defining a methodology for oral rehabilitation of edentulous patients. The design process is based on the definition of a reliable maxillofacial virtual model, which includes jawbones, dentition and oral soft tissues along with the relative position of vascular vessels and nerves. Moreover, a diagnostic prosthesis is included within the model to represent the desired outcome (final restoration). The implant position within the virtual model of a patient's mouth considers the available bone density, the distance from adjacent teeth and/or other implants, the fixtures proximity to sensory nerves (e.g., mandibular alveolar nerve), and the relationship with the prosthetic end result. The designed implant placements are transferred into the surgical field by means of a customized surgical guide. The surgical guide is produced through an additive manufacturing process and geometrically controlled before performing jawbone osteotomies. 
In this section, the proposed methodology is presented and the tools used to improve the accuracy of the outcome are described. The overall procedure can be summarized in the following steps:

1. creation of the 3D virtual maxillofacial model;

2. virtual design of the implant placement;

3. virtual design of the surgical guide;

4. production and testing of the surgical guide.

Figure 1 schematizes the overall workflow of the developed CAD/CAM framework for flapless dental implant surgery. The clinical protocol of this study was approved by the Ethics Committee at the University of L'Aquila Dental Clinic, and written form approval was obtained by the patients.

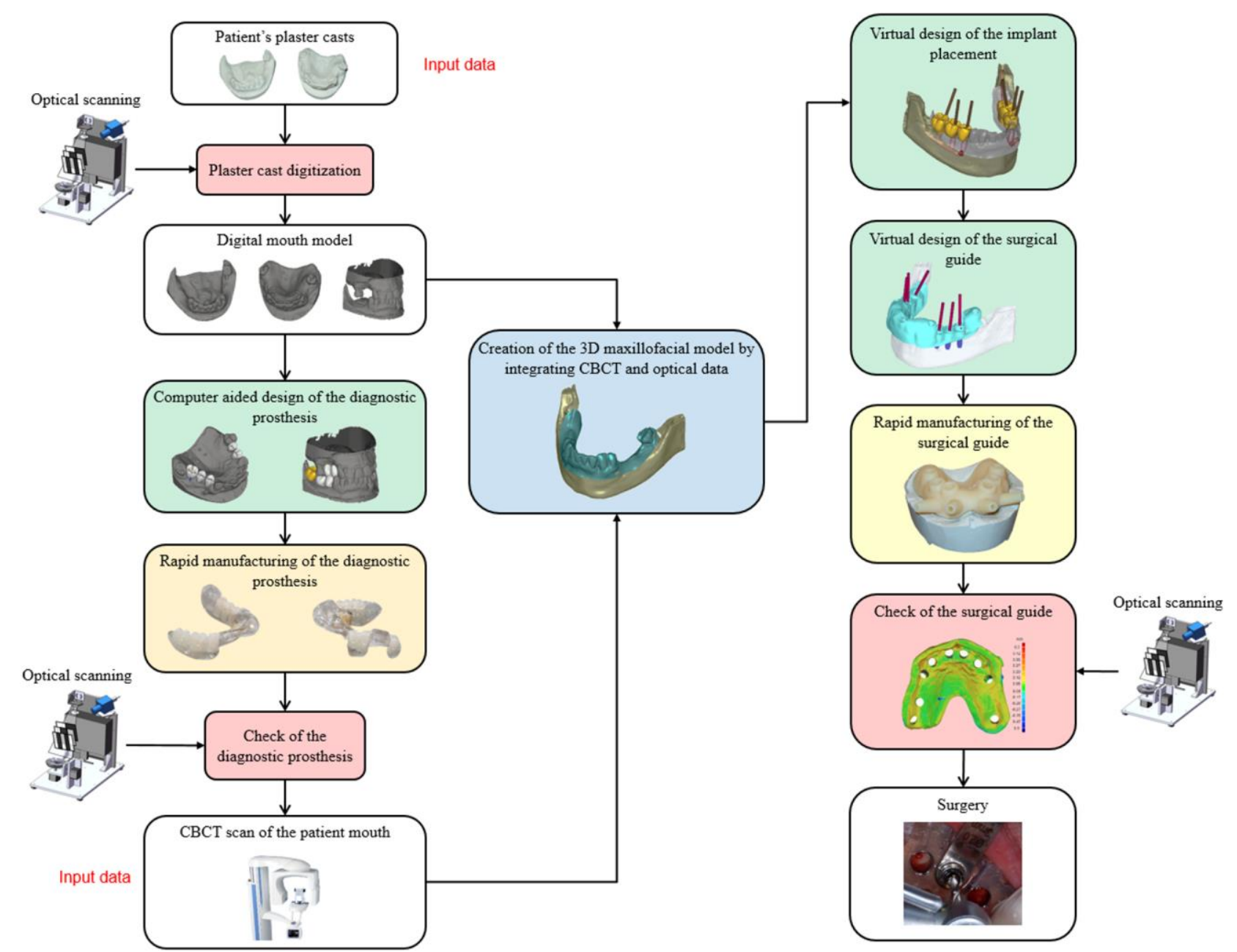

Figure 1 - Developed CAD/CAM framework for flapless dental implant surgery.

\subsection{Creation of the 3D virtual maxillofacial model}

The accurate creation of a 3D virtual anatomical model of the patient's mouth is the crucial step in dental implant surgery planning. In this paper, CBCT scanning and optical scanning are combined to capture the anatomical models. In particular, CBCT (Planmeca ProMax 3D) is used to reconstruct skeletal structures with lower radiation doses and shorter acquisition time with respect to conventional CT scanning [22-24]. 
Tomographic data do not provide mucosa soft tissues and dentition structures with accuracy as required for guided dental implant surgery. Moreover, the presence of streak artifacts owing to metal restorations may significantly obscure dental arches in the CBCT scan. These potential drawbacks are overcome by using an optical scanner to reconstruct accurate 3D models of patient's dentition and oral soft tissues. The non-contact nature of optical scanning technology allows for non-invasive and accurate 3D reconstructions of dental impressions or plaster models (digital mouth model). However, the use of optical scanners is limited to the acquisition of visible surfaces, whereas skeleton internal anatomies cannot be digitized $[18,19,25]$. The reliable creation of a virtual anatomical model, including jawbones, dentition and oral soft tissues, must then go through a fusion process in order to integrate anatomical data sets obtained by CBCT imaging and optical scanning. The captured data are combined into a unique frame by using common anatomical areas and/or radiographic templates as references for the matching process. In this context, the major challenge relies on obtaining multi-tissue models without losing accuracy. The overall process can be summarized by the following steps:

1. creation of a digital mouth model;

2. design and manufacturing of the diagnostic prosthesis;

3. CBCT recording of the maxillofacial region;

4. integration between CBCT and optical data.

\subsubsection{Digital mouth model}

The acquisition system has been specifically developed with the aim at digitizing both dental impressions and plaster casts (Figure 2-a). The optical unit is composed of a 8-bit monochrome CCD digital camera (The Imaging Source ${ }^{\circledR}$ DMK 41BF02, resolution $1280 \times 960$ pixels), equipped with a $16 \mathrm{~mm}$ focal length lens (PENTAX, C31634KP 2/3" C-mount), and a multimedia white light DLP ${ }^{\circledR}$ projector (OPTOMA EX330e, resolution XGA $1024 \times 768$ pixels), which are used as active devices for a triangulation process. A multitemporal Gray Code Phase Shift Profilometry (GCPSP) method is used for the 3D shape recovery through the projection of a sequence of black and white vertical fringes. The methodology is able to provide surface measurements within a working volume of $100 \mathrm{~mm} \times 80 \mathrm{~mm} \times 80 \mathrm{~mm}$ (width $\times$ height $\times$ depth), with a spatial resolution of $0.1 \mathrm{~mm}$ and an overall accuracy of $0.01 \mathrm{~mm}$ [26]. Final digital reproductions of patient's dental structures (digital mouth models) are obtained by merging different range maps within an automatic multiview scanning process (Figure 2-b).
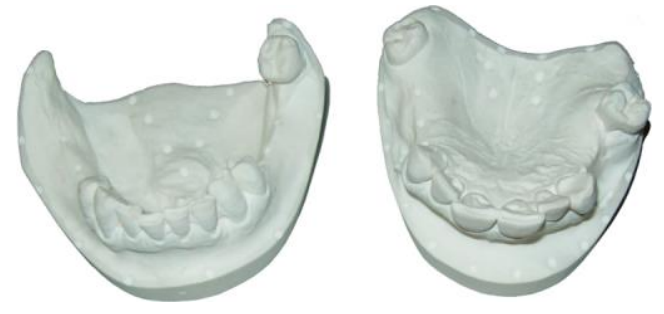

(a)
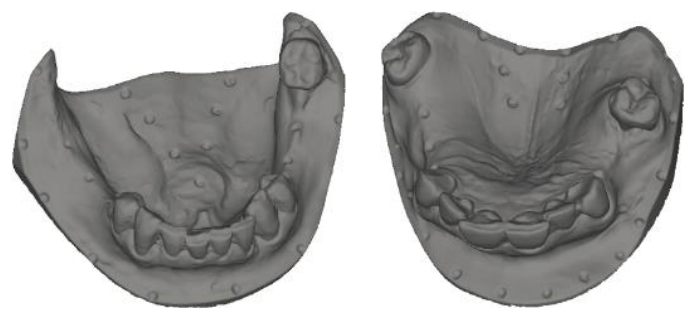

(b)

Figure 2 - Plaster casts (a) and corresponding digital reconstructions obtained by the optical scanner (b). 


\subsubsection{Design and manufacturing of the diagnostic prosthesis}

The aim of the diagnostic prosthesis is the reproduction of the final restoration satisfying both functional and aesthetical requirements. For fully or severe edentulous patients, the diagnostic prosthesis has to be used as reference in the fusion stage (see section 2.1.4). In these cases, patients have to wear the prosthesis during the tomographic data acquisition.

A CAD software [27], specific for dental restoration, is used. Some virtual tools allows for verifying geometrical and functional constraints, guiding the technician during the prosthesis design in terms of shape and position. In this context, tooth orientations can be interactively varied by shifting or tilting movements, and the dimensions can be adapted with regard to the available prosthetic space and/or the required occlusion condition (Figure 3).

Diagnostic prostheses are manufactured by rapid prototyping the designed virtual models (Figure 4-a,b). In this work, an additive prototyping methodology was used (Objet Eden500 from Stratasys ${ }^{\circledR}$ ). The prototype is made by a bio-compatible material (Objet Med610), which presents a great dimensional stability and a colourless transparency. This material has five medical approvals including Cytotoxicity, Genotoxicity, Delayed Type Hypersensitivity, Irritation and USP Plastic Class VI.

The design and manufacturing of diagnostic prostheses are verified by fitting the templates onto the plaster models (Figure 5). Technicians can still slightly refine the prosthesis if aesthetical changes or adjustments of the mating surfaces are required. In these cases, diagnostic prostheses have to be further acquired by the optical scanner in order to recreate digital models to be used for downstream processes (Figure 4-c).
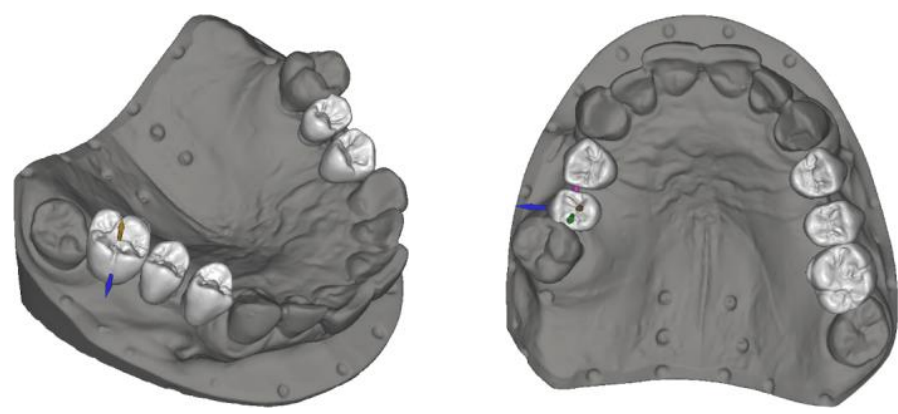

(a)

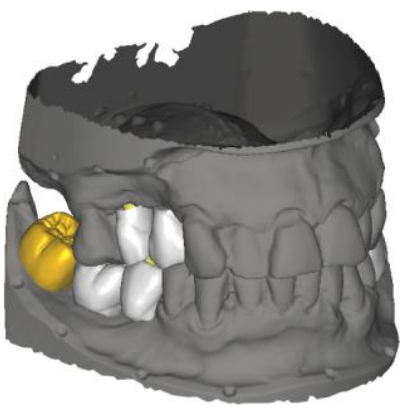

(b)

Figure 3 - Virtual design of the restorative elements of a diagnostic prosthesis directly using the digital reconstruction of plaster samples (a). Occlusion conditions can be simulated in order to verify the proper placements of prosthetic elements (b).

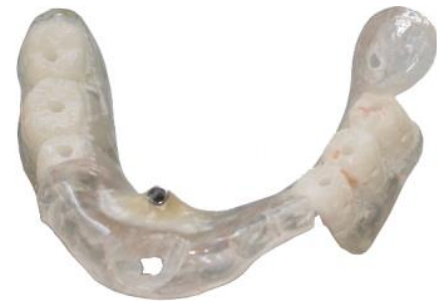

(a)

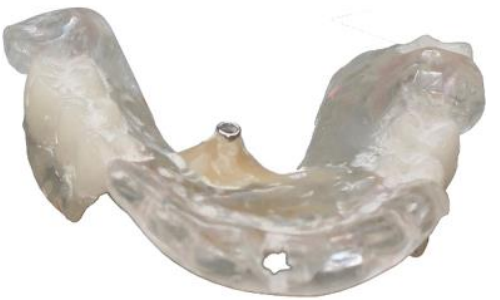

(b)

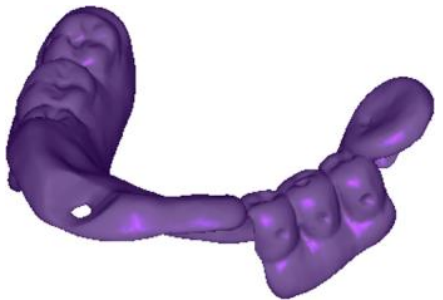

(c)

Figure 4 - Manufactured diagnostic prostheses for superior (a), and inferior (b) arches. 3D digital model obtained by the optical scanner (c). 


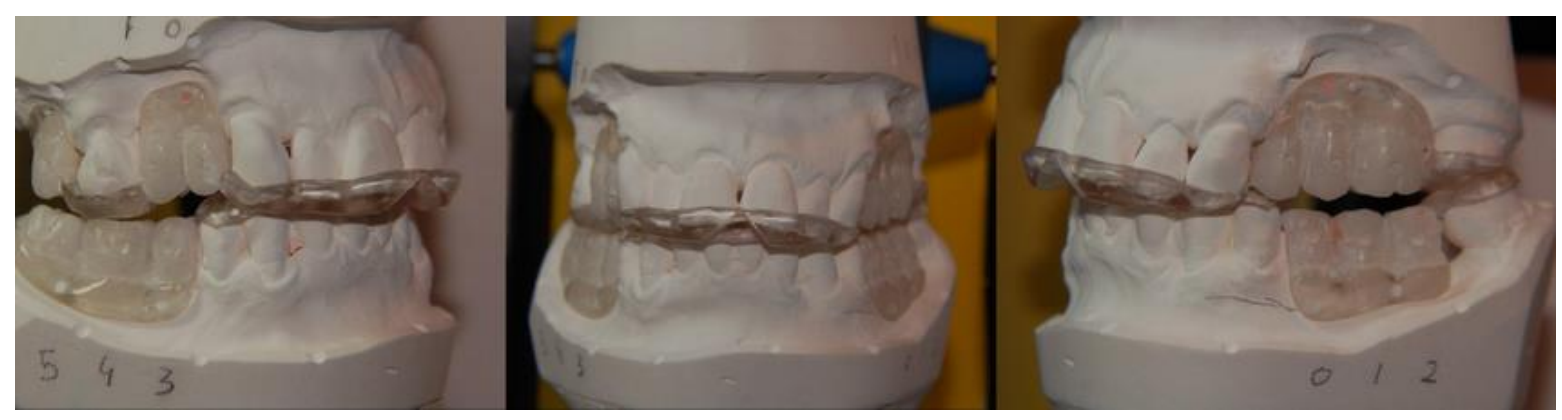

Figure 5 - Fitting of the manufactured templates verified onto the plaster models.

\subsubsection{CBCT recording of the maxillofacial region}

CBCT data are stored in DICOM (Digital Imaging and Communications in Medicine) images and processed to generate volumetric representations of anatomical tissues. However, the accuracy of 3D models derived from $\mathrm{CBCT}$ data is greatly influenced by acquisition parameters and reconstruction settings [12]. In particular, a volumetric representation is typically the result of a user-entered threshold value based on visually segmenting different tissues. Robustness and efficiency of the segmentation process are affected by many factors: image contrast, image noise and prior knowledge about anatomies. In this paper, a "reference thresholding" method has been developed for the reconstruction of 3D models including bone structures and dentitions. DICOM images are segmented by using 3D models obtained by optically scanning dental plaster models as ground truth ("reference") of the CBCT reconstruction process. The optimal threshold value is computed in order to minimize the differences between the digital models obtained by the two different scanning technologies.

\subsubsection{Integration between CBCT and optical data}

The fusion between bone structures (CBCT data) and dentition models (optical scan data) is operatively obtained by following two different approaches, which depend on the edentulism classification:

- minimally compromised edentulous mouth: the number of natural teeth is sufficient to integrate the dental model reconstructed by optically scanning the plaster sample within CBCT data on the basis of a best fitting alignment (Figure 6);

- severe or totally compromised edentulous mouth: an intraoral radiographic template (diagnostic prosthesis containing radiopaque agent, e.g., barium sulfate) is used during the CBCT patient scan (Figure 7).

The first approach is carried out by segmenting CBCT teeth using an optimal threshold value, which minimize 3D discrepancies when multi-modal dentition models are aligned [28]. This approach is based on a markerfree registration which uses tooth crown shapes as registration references. The methodology consists in the 3D crown reconstruction by segmenting CBCT images through different threshold values. The optimal threshold value is then determined by using the optically-scanned crown model as ground truth. In particular; the optically-scanned crown model is imported within the DICOM volume data set and aligned to each different 
model corresponding to a specific threshold value. The alignment is performed by manually identifying at least three common points and refined by point-based best fitting techniques. Mating surfaces to be used in the refinement of the alignment process are identified within a distance value $\left(\delta_{\varepsilon}=1 \mathrm{~mm}\right)$ in order to avoid misalignments due to noisy data. An optimal threshold $\left(\tau_{o p t}\right)$ is finally computed as the value which minimizes the discrepancies between the overlapping crown tissues, reconstructed by the optical and the CBCT imaging technologies.

The second approach must be carried out when the natural dentition of the patient is not sufficient to guarantee an accurate reference for the alignment process. In these cases, radiopaque geometries are incorporated within the prototyped diagnostic prosthesis (e.g., radiopaque markers as shown in Figure 7-a, or entire radiopaque geometries as the prosthetic crown surfaces shown in Figure 12). Radiopaque surfaces can then be reconstructed from the CBCT scan (Figure 7-a) and used as reference surfaces to integrate the optical scan within the tomographic model (Figure 7-b) by using the same methodology adopted for partially edentulous patients.

The two approaches only differ in the material used for the geometries (e.g., crowns) used as reference for the matching process. However, the radiopaque material does not affect the accuracy of the optical scanning results. Moreover, the $\mathrm{CBCT}$ reconstruction of the radiopaque prostheses always gives better results with respect to natural dentition due the intrinsic uniformity of the scanned material (certain lack of metallic dental fillings).

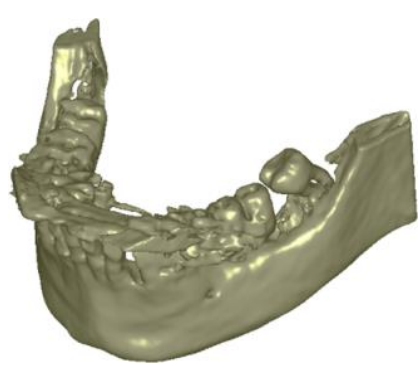

(a)

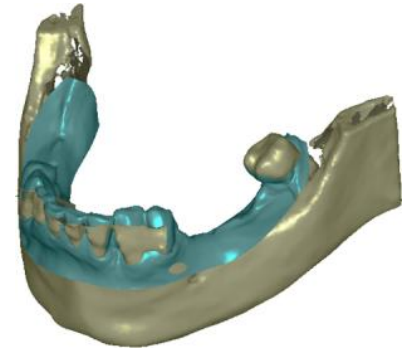

(b)

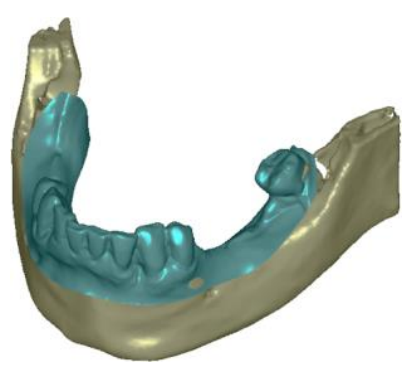

(c)

Figure 6 - CBCT jawbone model presenting artefacts (a), alignment with the digital mouth model obtained by the optical scanner (b) and final integration between CBCT and optical data after artefacts removal.

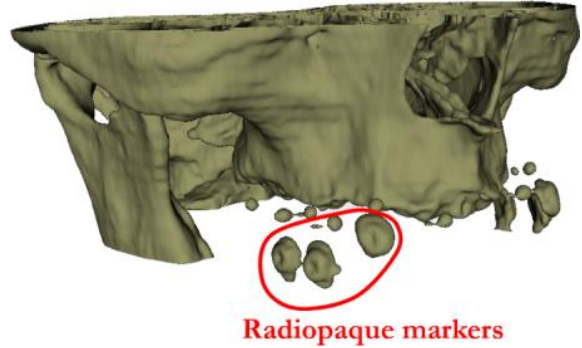

(a)

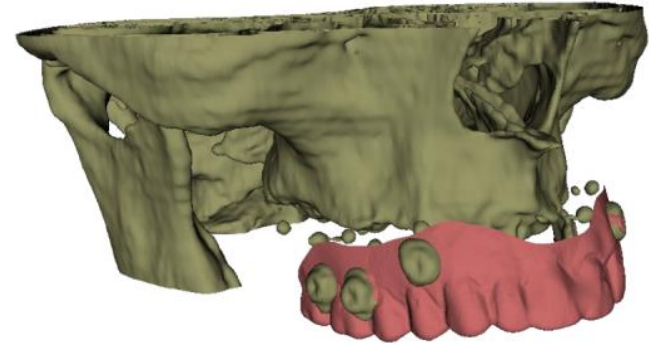

(b)

Figure 7 - Radiopaque markers, incorporated within the acrylic resin of the diagnostic prosthesis, and reconstructed from the CBCT scan (a), integration between the digital model of the diagnostic prostheses and the CBCT jawbone reconstruction (b). 


\subsection{Virtual design of the implant placement}

The functionality of a dental prosthesis with respect to biomechanical and aesthetical characteristics requires a well-designed implant positioning. The ideal fixture loading should be only compressive axial, with no lateral forces involved. Lateral components of the occlusal loading conditions cause bending stresses which compromise long-term implant stability, in particular with regard to the crestal bone resorption. Moreover, the implant vertical axis should fall in the middle of the perimeter identified by the cuspidal crests of the tooth occlusal plane [29]. Fixtures should be placed at least $1.5 \mathrm{~mm}$ far from adjacent teeth in order to avoid loss of the interproximal height of bone and papilla [30,31]. Moreover, adequate access for oral hygiene and correct gingival contour requires a bone thickness in the vestibular-palatal dimension at least 2-3 $\mathrm{mm}$ greater than the implant diameter, even if there are no sufficient evidences to establish with absolute confidence the minimum vestibular bone thickness for an optimal aesthetic outcome [32]. The papilla is defined to be present when it fills the entire interproximal space or part of this space. A $3 \mathrm{~mm}$ implant-to-implant distance has demonstrated to provide an adequate papillary fill $[33,34]$.

The methodology proposed in this work simplifies the analysis of the prosthetic space with regard to the fixtures positioning. The accurate assessment of both hard and soft perimplantar tissues allows the determination of the available prosthetic space which is needed during the design of the prosthesis and choice of implant system and prosthetic components.

The final model is composed of the patient's jawbone structure, oral soft tissues and existent dentition with the fixtures placed within bone (Figure 8-a).

\subsection{Virtual design of the surgical guide}

The surgical guide must provide an accurate transfer of the planned surgery from the virtual model to physical one. The main step of the surgery consists in drilling the soft tissue together with the hard tissue, and can be carried out with subsequent steps varying the drilling diameter. These phases should be done in a comfortable way for the patient and the surgeon. Moreover the surgeon must be confident with the physical framework, which should provide accuracy and reliability to assure the success of the surgery within short operative times. The surgical guide must guarantee a perfect fit on existing dentition and soft tissues along with a structural stability during the drilling phases. For this reason, the surgical guide is directly modelled on the final virtual model within the CAD environment [27]. A uniform layer of material is added to the reference surface of the virtual mouth and a shell of a specific depth is created. Then, the guide must be completed with sleeves supporting drilling (Figure 8-b). Starting from the designed position of the implants, cylinders of a specific diameter and holes for the metal sleeves are added to the modelled shell. The cylinders can be designed with different heights, according to planned fixtures length and depth, in order to achieve a drilling depth stop. If needed (e.g, for mucosa-supported guides), the guide can be anchored with horizontal stabilizing pins (locking system). Their position is planned by the surgeon and the dental technician during the design phase and their placement is guided through additional sleeves. 


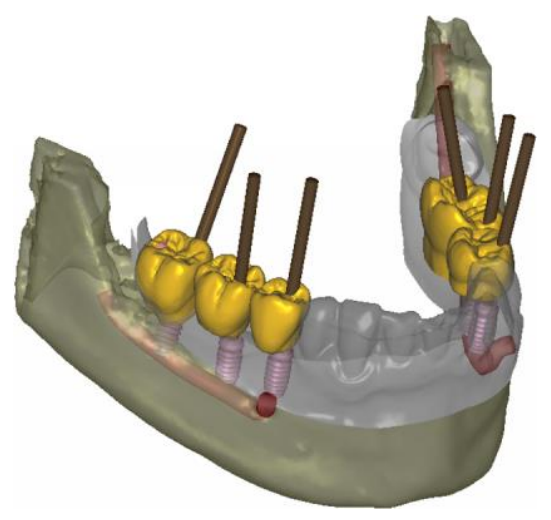

(a)

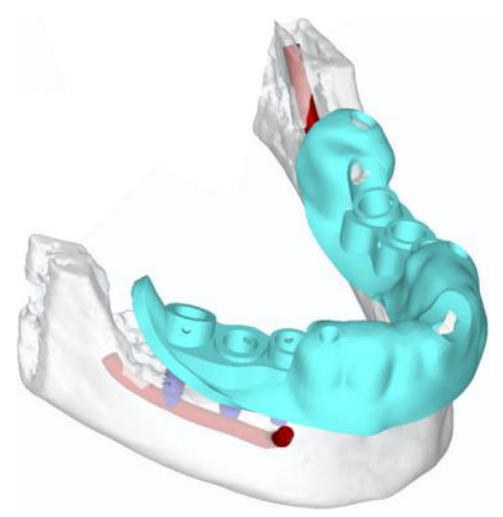

(b)

Figure 8 - Patient's jawbone structure with oral soft tissues and existent dentition with designed fixtures placed within bone (a), design of the surgical guide on the maxillofacial model (b).

\subsection{Production and testing of the surgical guide}

The surgical guide is manufactured by additive manufacturing processes (see section 2.1.2). The compliance of the final template is verified by comparing the designed and the manufactured shapes. The manufactured shape is reconstructed by using the optical scanner and overlapped to the CAD model in order to evidence existing geometrical deviations (Figure 9-a). Moreover, the accurate fitting of the guide with the original plaster dental model is manually checked (Figure 9-b). Possible discrepancies between the mating surfaces of the different models may result in improper fitting of the surgical guide, thus turning out to be not wearable (undercut surfaces) or instable in the patient's mouth.

Drilling sleeves are then inserted and glued in the template, which represents the actual guiding system for the surgeon during operative step. Therefore, a final check is directly carried out into the patient's mouth in order to verify the proper fitting onto the dento-alveolar arch, where soft tissues are present.

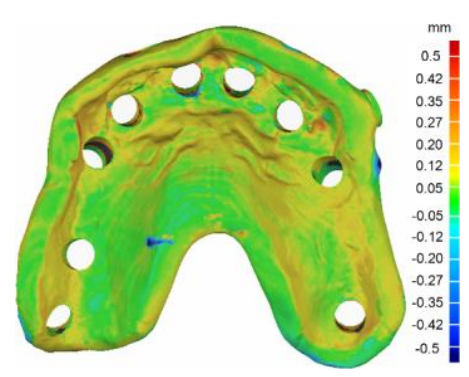

(a)

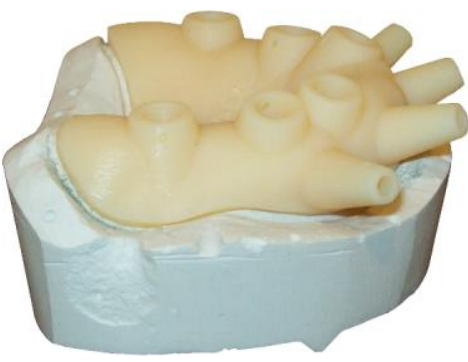

(b)

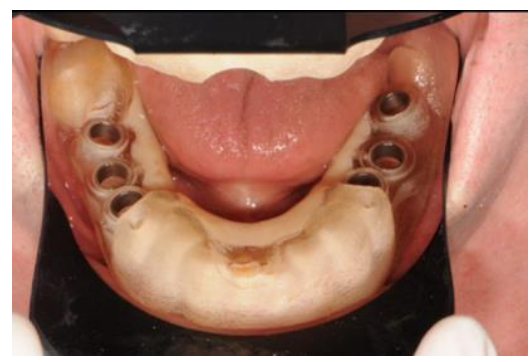

(c)

Figure 9 - Comparison between designed and manufactured shapes of the surgical guide (a), guide fitting on the original plaster dental model (b), final check directly carried out into the patient's mouth (c). 
The CAD-CAM framework has been experienced in clinical practices. The results regarding two clinical cases (a fully edentulous and a partial edentulous patient) are reported along with the description of flapless treatments adopted in order to insert a total of 11 implants.

The surgical guides were treated in TAED solution in order to obtain a chemical sterilization for two hours, then placed within the patients' mouths and fixed with horizontal stabilizing pins.

Osteotomies were accomplished in correspondence of metallic sleeves inserted within the guiding template accordingly to the planned fixtures positions and angulations. A sequence of different drill guides (keys) with different inner diameters, from the smallest to the largest, were used with increasing twist drill diameters. This allowed final drill sizes with the maximum accuracy. The drilling sequences were executed for each implant starting from the first drill through the guide $(2 \mathrm{~mm}$ diameter pilot drill) to the final implant insertions. Moreover, the sleeves height provided the drilling stop for the proper drill depth. All the implants achieved excellent primary implant stability (final torque exceeding $30 \mathrm{Ncm}$ ), according to the requirements for longterm success with immediate-loading implants.

When all the sites were prepared, the surgical guides were removed and healing abutments and/or immediate loading prostheses were fixed onto the fixtures inserted within jawbones.

\subsection{Fully edentulous patient}

The patient, a 52-years-old man, presented a fully edentulous upper jaw (Figure 10). A full-arch replacement plan with dental implants was selected to treat the edentulism. The desired prosthetic outcome was modelled on the 3D digital mouth model by using the CAD platform (Figure 11-a,b). The diagnostic prosthesis was manufactured by the rapid prototyping technique using two materials: a transparent photopolymer for the soft tissue (Objet Med610) and a radiopaque agent for the teeth. The manufactured template was firstly checked onto the plaster model (Figure 11-c) and then directly into the patient's mouth in order to verify both fitting and aesthetical outcomes.

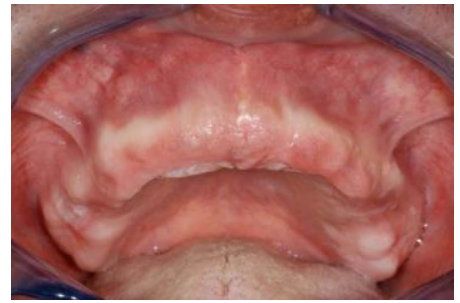

(a)

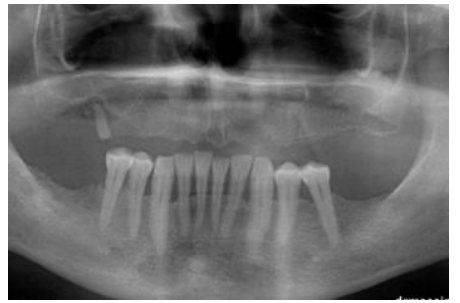

(b)

Figure 10 - Fully edentulous patient: picture of the jaw (a) and relative panoramic radiograph (b). 


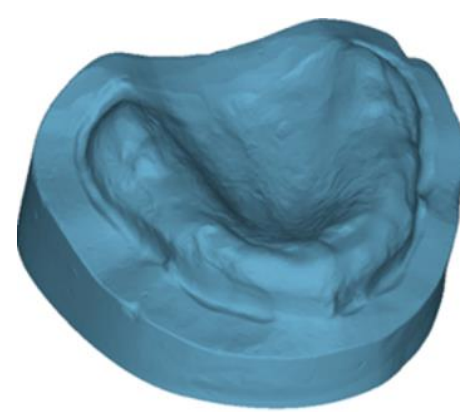

(a)

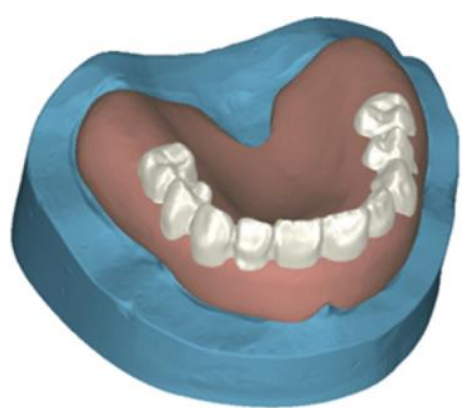

(b)

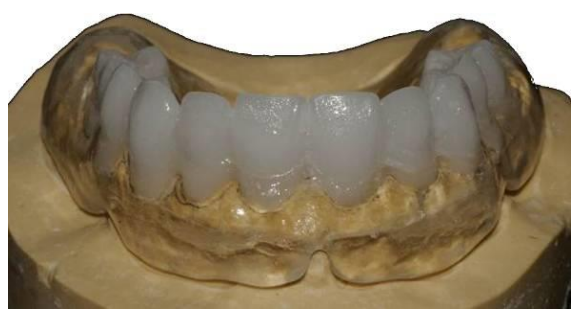

(c)

Figure 11 - Edentulous jaw's digital mouth model (a) and diagnostic prosthesis directly modelled on the 3D digital mouth model (b), checking of the manufactured diagnostic prosthesis by fitting on the plaster model (c).

A CBCT scan with the patient wearing the diagnostic prosthesis was done. Optical and CBCT data were geometrically matched superimposing radiopaque reference surfaces and optimal CBCT data threshold value was achieved (Figure 12).

The implant placement was directly designed by the surgeon using dedicated software taking into account anatomical, biomechanical and aesthetical requirements. A total of 7 implants and 3 transalveolar horizontal stabilizing pins were virtually inserted within the integrated 3D data model (Figure 13).

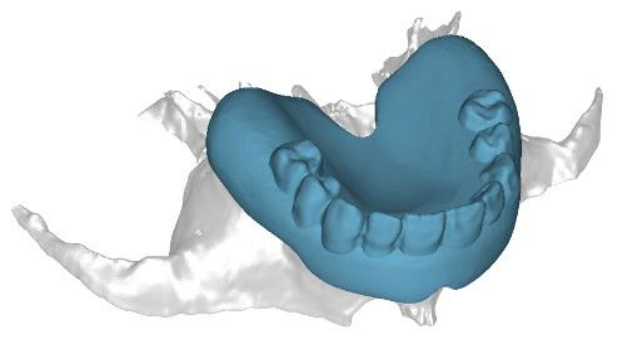

(a)

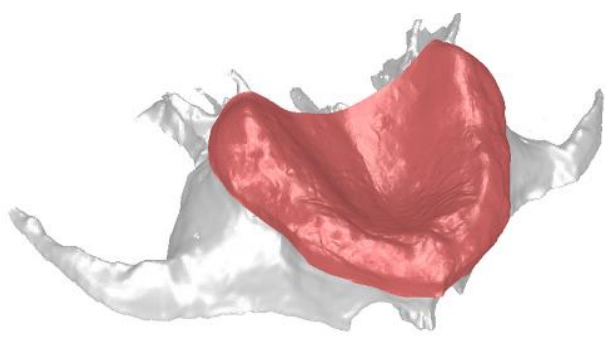

(b)

Figure 12 - Fusion of optical and CBCT scanning data (b) by means of the diagnostic prosthesis (a).

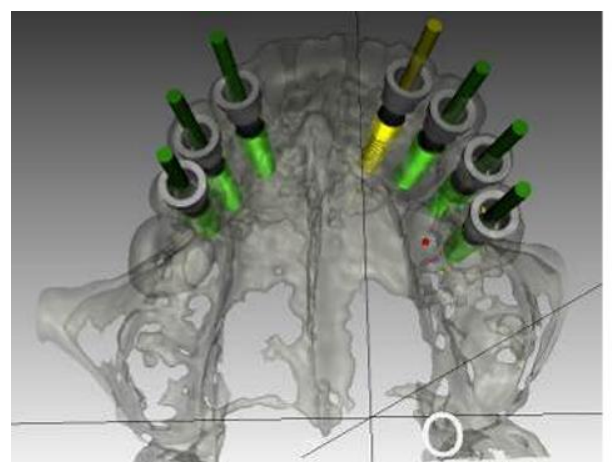

(a)

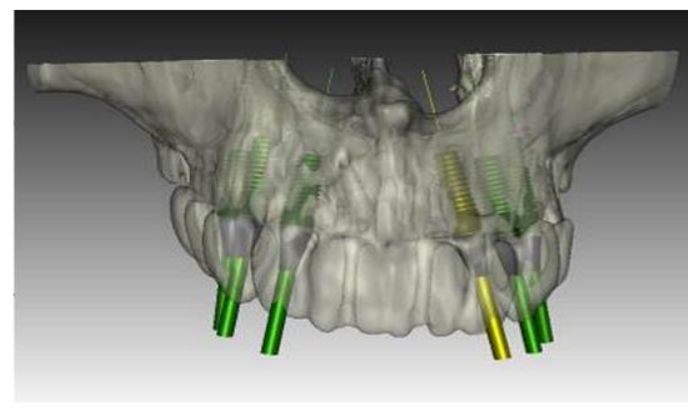

(b)

Figure 13 - Computer aided design of implant placement within the 3D maxillofacial virtual model.

The following step consisted in designing and manufacturing the surgical guide on the basis of the data extracted from the planning software (Figure 14). The accuracy of the manufactured surgical guide was 
verified by comparing the $3 \mathrm{D}$ reconstruction obtained by the optical scanner with the planned model (Figure $15)$.

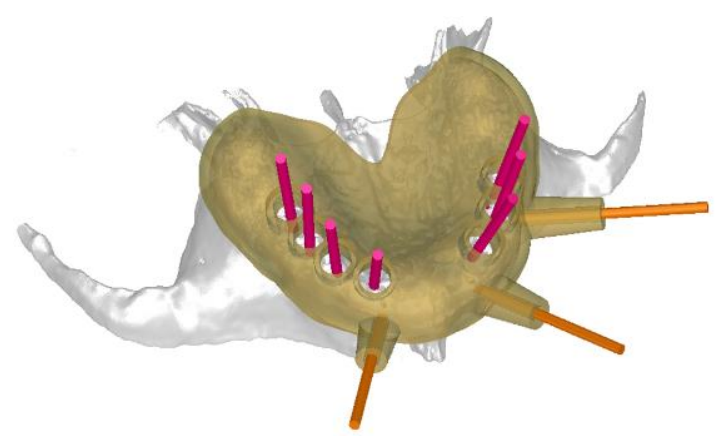

(a)

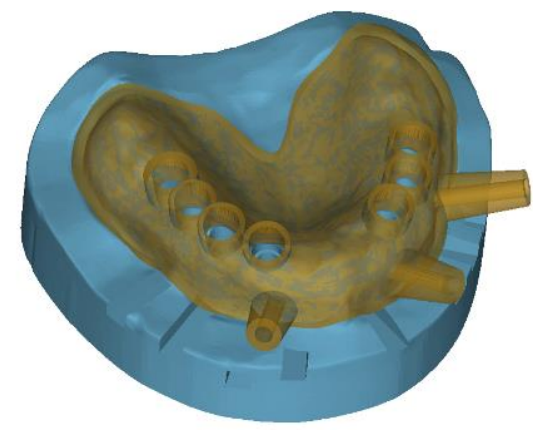

(b)

Figure 14 - Surgical guide modelled on the 3D virtual model and on the digital mouth model.

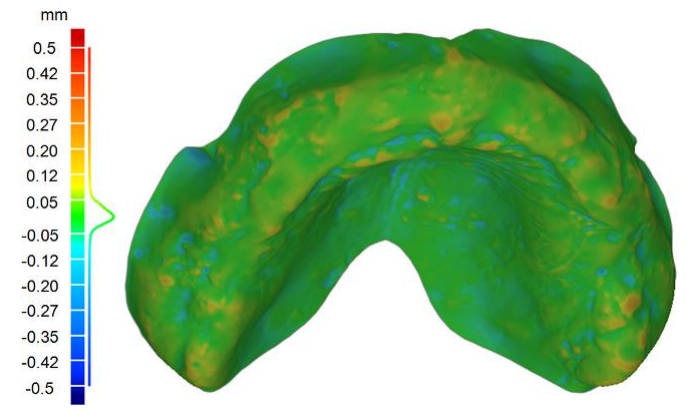

Figure 15 - 3D compare between designed and manufactured shapes of the surgical guide.

The planned implant position, angulation and depth, was guaranteed by the flapless guided surgery (Figure 16). Immediately after implant placement (Figure 17-a), the abutments were placed on the fixtures and the provisional pre-fabricated prosthesis checked. Final preparation of the inner surface of the prosthesis was performed using an acrylic resin in order to achieve a perfect fit on the abutments. The prosthesis was then unscrewed, resin in excess was carefully removed, and finally inserted and screw-retained during the same surgical appointment. (Figure 17-b).

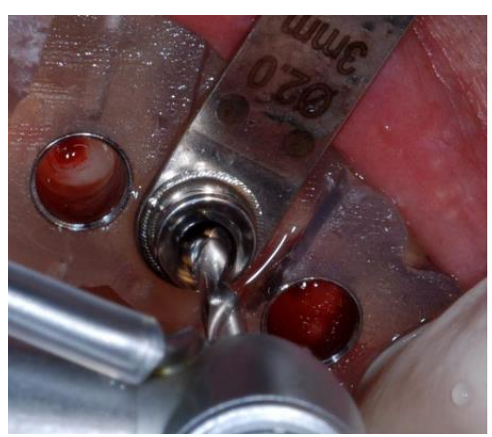

(a)

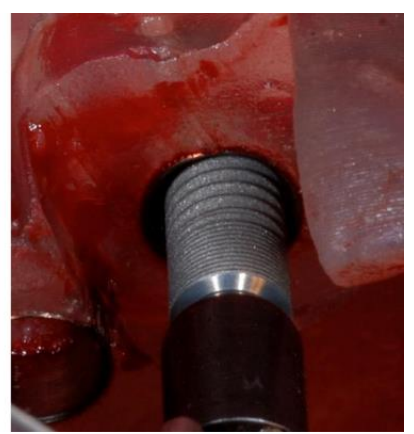

(b)

Figure 16 - Flapless drilling of the bone through mechanical sleeves (a) and insertion of the implants (b). 


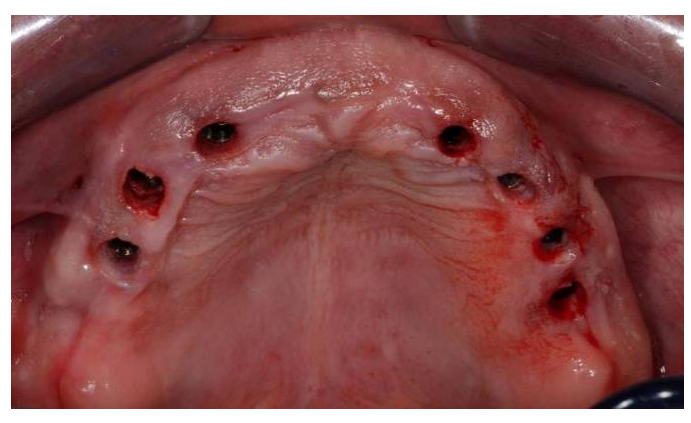

(a)

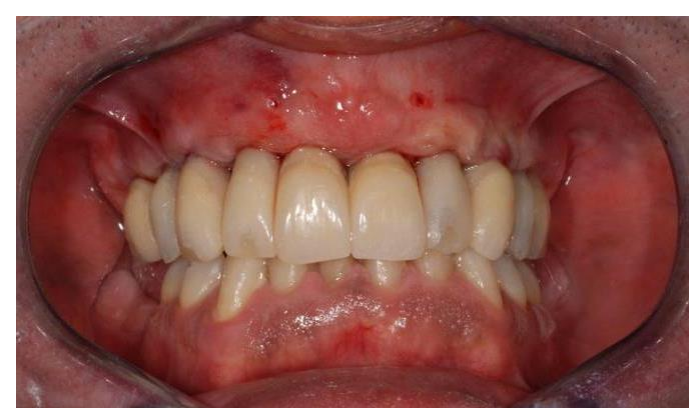

(b)

Figure 17 - Postoperative intraoral view (a) and prosthesis for immediate loading (b).

\subsection{Partially edentulous patient}

A 44-years old partially edentulous patient was referred for a dental implant restoration. The virtual model was created by a digital mouth model and the ideal prosthetic outcome was virtually designed by the CAD software (Figure 18-a). In this case of partially edentulous area the CBCT was done without the diagnostic prosthesis. The different scanning data were integrated by matching reference surfaces from the existing dentition structure. 4 implants and 2 transalveolar horizontal stabilizing pins were planned (Figure 18-b) and a rapid prototyping surgical guide was designed and produced (Figure 19).

The implants were inserted using a totally guided flapless surgery approach (Figure 20-a). Immediately after surgery, the temporary restoration was screwed on the fixtures (Figure 20-b).

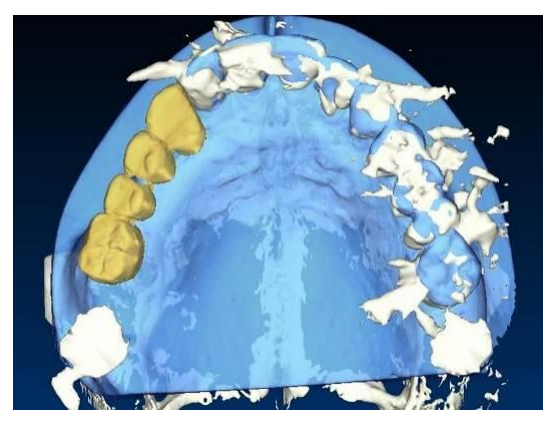

(a)

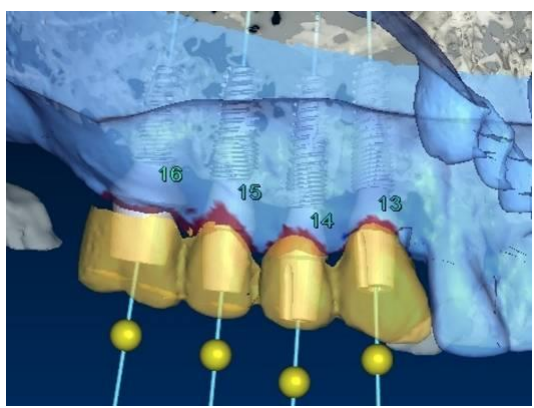

(b)

Figure 18 - Integration between optical and CBCT data along with the design of the desired prosthetic outcome (a), virtual implant placement based on the desired prosthetic outcome (b).

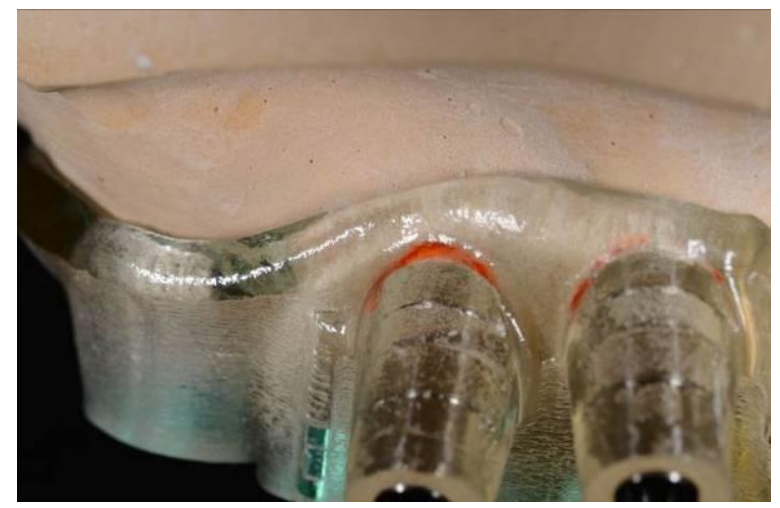

Figure 19 - Detail of the manufactured surgical guide fitted on the dental plaster model. 


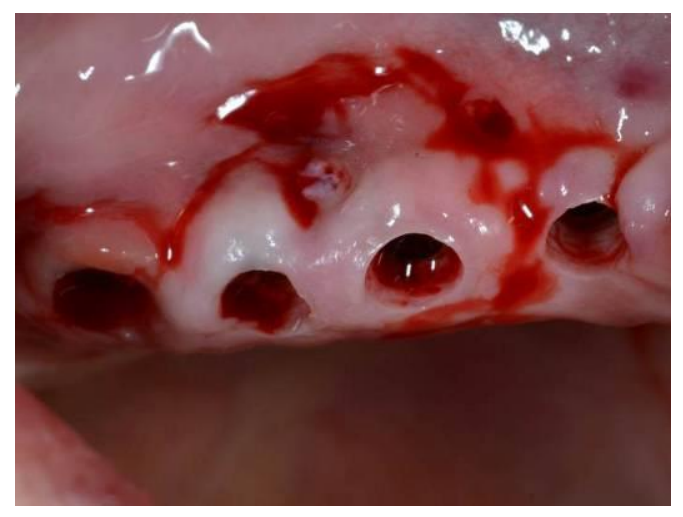

(a)

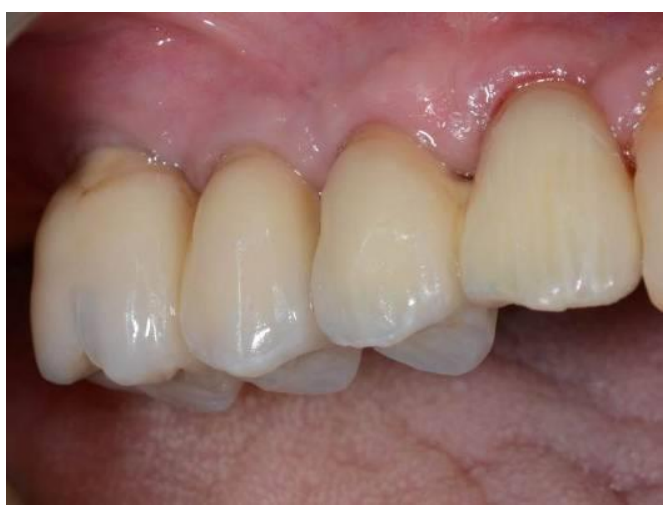

(b)

Figure 20 - Postoperative intraoral view (a) and screwed four-abutment splinted prosthesis for immediate loading after the surgery (b).

\section{Conclusions}

The presented CAD-CAM framework aims at minimizing error sources in the fabrication of surgical guides to be used in the fixtures positioning process. An optical scanning technology based on a structured lighting approach is used in conjunction with the traditional CBCT scan based preoperative planning process. The use of the optical scanner, which provides higher resolution and accuracy than CBCT scanning, allows the reconstruction of reliable dentition models with associated soft tissues. These models are used to optimize the segmentation process of DICOM images, contextually cleaning up image artifacts and tissues not belonging to the target structure. The fusion between the optically-scanned crown model and the CBCT crown volume allows for the automatic removal of artifacts, generated by dental fillings and/or orthodontic fixed appliances, thus retrieving the real dental patient anatomy. Final CBCT dentition anatomies are only used as references to fuse dentition models obtained by optically scanning plaster samples, and jawbones reconstructed from CBCT data. This procedure guarantees an accurate placement of crown shapes and oral soft tissues, captured by the optical scanner, with respect to the CBCT alveolar bone structure, avoiding subjective visual perceptions during the reconstruction process. Moreover, when partially edentulous patients are involved, the production of radiographic templates is not needed, since the natural dentition can be used as the reference area to integrate the scanned data.

The accurate reconstruction of oral surfaces represents a valid support in the management of soft tissues and residual dental elements during the planning phase, as well as the outcome predictability when flapless approaches are carried out. Optical scanning has demonstrated to be particularly effective in evaluating the accuracy of the various physical models adopted in the treatment planning process (plaster model, diagnostic prosthesis, surgical guide), thus allowing to point out possible error sources. A fully digitized workflow aims at reducing geometrical inaccuracies related to skilful human effort, avoiding any laboratorial step in implant planning stage. Moreover, the design phase of patient fitting templates (i.e., diagnostic prosthesis, surgical guide) and the planning process of implants positioning greatly benefit from a continuous interaction between the clinician's knowledge and the anatomical as well as geometrical attributes. 
Clinical cases have been carried out in order to test the proposed methodology. The reproducibility and high precision of the proposed technique has enabled the prosthodontic-driven placement of immediate loading implants with flapless surgery protocols. Shorter surgery and treatment times, mini-invasive surgical technique and the immediate use of a fixed prosthesis were the main items involved in the reported clinical cases. The flapless protocol enhanced the prosthodontic-driven placement of implants using safe and monitored surgery approach, improving patient care and reducing the likelihood of unpredictable and undesirable outcomes. Nevertheless, its correct management still requires an unavoidable surgical and prosthetic clinical experience.

\section{Competing interests}

The authors declare no competing interests.

\section{Informed consent documentation}

Written informed consent was obtained from the patients for publication of the presented case reports and any accompanying images. A copy of the written consent is available for review by the Editor-in-Chief of this journal.

\section{References}

1. Holst, S., Blatz, M.B., Eitner, S.: Precision for computer-guided implant placement: Using 3D planning software and fixed intraoral reference points. J Oral Maxil Surg 65(3), 393-399 (2007). doi:DOI 10.1016/j.joms.2006.10.050

2. Bedi, A., Michalakis, K.X., Mariani, E.J., Zourdos, D.M.: Immediately Loaded Maxillary and Mandibular Dental Implants with Fixed CAD/CAM Prostheses Using a Flapless Surgical Approach: A Clinical Report. J Prosthodont 20(4), 319-325 (2011). doi:DOI 10.1111/j.1532-849X.2011.00689.x

3. Sclar, A.G.: Guidelines for flapless surgery. J Oral Maxil Surg 65(7), 20-32 (2007). doi:DOI 10.1016/j.joms.2007.03.017

4. Azari, A., Nikzad, S.: Flapless implant surgery: Review of the literature and report of 2 cases with computer-guided surgical approach. J Oral Maxil Surg 66(5), 1015-1021 (2008). doi:DOI 10.1016/j.joms.2007.12.010

5. Nikzad, S., Azari, A.: Custom-Made Radiographic Template, Computed Tomography, and ComputerAssisted Flapless Surgery for Treatment Planning in Partially Edentulous Patients: A Prospective 12Month Study. J Oral Maxil Surg 68(6), 1353-1359 (2010). doi:DOI 10.1016/j.joms.2009.04.108

6. Rossi, R., Morales, R.S., Frascaria, M., Benzi, R., Squadrito, N.: Planning implants in the esthetic zone using a new implant 3D navigation system. The European journal of esthetic dentistry : official journal of the European Academy of Esthetic Dentistry 5(2), 172-188 (2010).

7. Abboud, M., Orentlicher, G.: An Open System Approach for Surgical Guide Production. J Oral Maxil Surg 69(12), E519-E524 (2011). doi:DOI 10.1016/j.joms.2011.07.027

8. Meloni, S.M., De Riu, G., Pisano, M., Cattina, G., Tullio, A.: Implant treatment software planning and guided flapless surgery with immediate provisional prosthesis delivery in the fully edentulous maxilla. A retrospective analysis of 15 consecutively treated patients. Eur J Oral Implantol 3(3), 245-251 (2010).

9. Maio, P., de Araujo Nobre, M., Lopes, A.: The use of computer-guided flapless implant surgery and four implants placed in immediate function to support a fixed denture: Preliminary results after a mean follow-up period of thirteen months. J Prosthet Dent 97(6), S26-S34 (2007).

10. Marchack, C.B.: An immediately loaded CAD/CAM-guided definitive prosthesis: A clinical report. J Prosthet Dent 93(1), 8-12 (2005). doi:DOI 10.1016/j.prosdent.2004.10.012 
11. Frascaria, M., Casinelli, M., Marzo, G., Gatto, R., Baldi, M., D'Amario, M.: Digital implant planning for a minimally invasive surgery approach: a case letter of a full-arch rehabilitation. Journal of Oral Implantology (2013). doi:10.1563/aaid-joi-d-12-00232

12. Guerrero, M.E., Jacobs, R., Loubele, M., Schutyser, F., Suetens, P., van Steenberghe, D.: State-of-the-art on cone beam CT imaging for preoperative planning of implant placement. Clin Oral Invest 10(1), 17 (2006). doi:DOI 10.1007/s00784-005-0031-2

13. Van Assche, N., van Steenberghe, D., Quirynen, M., Jacobs, R.: Accuracy assessment of computerassisted flapless implant placement in partial edentulism. J Clin Periodontol 37(4), 398-403 (2010). doi:DOI 10.1111/j.1600-051X.2010.01535.x

14. Van Assche, N., van Steenberghe, D., Guerrero, M.E., Hirsch, E., Schutyser, F., Quirynen, M., Jacobs, R.: Accuracy of implant placement based on pre-surgical planning of three-dimensional cone-beam images: a pilot study. J Clin Periodontol 34(9), 816-821 (2007). doi:DOI 10.1111/j.1600051X.2007.01110.X

15. Vercruyssen, M., Jacobs, R., Van Assche, N., Van Steenberghe, D.: The use of CT scan based planning for oral rehabilitation by means of implants and its transfer to the surgical field: a critical review on accuracy. J Oral Rehabil 35(6), 454-474 (2008). doi:DOI 10.1111/j.1365-2842.2007.01816.x

16. Neugebauer, J., Kistler, F., Kistler, S., Züdorf, G., Freyer, D., Ritter, L., Dreiseidler, T., Kusch, J., Zöller, J.E.: CAD/CAM-produced surgical guides: Optimizing the treatment workflow. International journal of computerized dentistry 14(2), 93-103 (2011).

17. D'haese, J., Van De Velde, T., Komiyama, A., Hultin, M., De Bruyn, H.: Accuracy and Complications Using Computer-Designed Stereolithographic Surgical Guides for Oral Rehabilitation by Means of Dental Implants: A Review of the Literature. Clin Implant Dent R 14(3), 321-335 (2012). doi:DOI 10.1111/j.1708-8208.2010.00275.x

18. M., v.d.Z.J.: Implant planning and placement using optical scanning and cone beam CT technology. $\mathrm{Br}$ Dent J 206(1), 23-23 (2009).

19. Bentz, R.M., Balshi, S.F.: Complete Oral Rehabilitation With Implants Using CAD/CAM Technology, Stereolithography, and Conoscopic Holography. Implant Dent 21(1), 8-12 (2012). doi:Doi 10.1097/Id.0b013e318243a1aa

20. Rosenfeld, A.L., Mandelaris, G.A., Tardieu, P.B.: Prosthetically directed implant placement using computer software to ensure precise placement and predictable prosthetic outcomes. Part 1: Diagnostics, imaging, and collaborative accountability. Int J Periodont Rest 26(3), 215-221 (2006).

21. Frisardi, G., Chessa, G., Barone, S., Paoli, A., Razionale, A., Frisardi, F.: Integration of 3D anatomical data obtained by CT imaging and 3D optical scanning for computer aided implant surgery. BMC Med Imaging 11 (2011). doi:Doi 10.1186/1471-2342-11-5

22. De Vos, W., Casselman, J., Swennen, G.R.J.: Cone-beam computerized tomography (CBCT) imaging of the oral and maxillofacial region: A systematic review of the literature. Int J Oral Max Surg 38(6), 609-625 (2009). doi:DOI 10.1016/j.ijom.2009.02.028

23. Miracle, A.C., Mukherji, S.K.: Conebeam CT of the Head and Neck, Part 1: Physical Principles. Am J Neuroradiol 30(6), 1088-1095 (2009). doi:Doi 10.3174/Ajnr.A1653

24. Miracle, A.C., Mukherji, S.K.: Conebeam CT of the Head and Neck, Part 2: Clinical Applications. Am J Neuroradiol 30(7), 1285-1292 (2009). doi:Doi 10.3174/Ajnr.A1654

25. Giordano, M., Ausiello, P., Martorelli, M.: Accuracy evaluation of surgical guides in implant dentistry by non-contact reverse engineering techniques. Dent Mater 28(9), E178-E185 (2012). doi:DOI 10.1016/j.dental.2012.06.006

26. Barone, S., Paoli, A., Razionale, A.V.: Computer-aided modelling of three-dimensional maxillofacial tissues through multi-modal imaging. Proceedings of the Institution of Mechanical Engineers, Part H: Journal of Engineering in Medicine 227(2), 89-104 (2013). doi:10.1177/0954411912463869

27. exocad GmbH. www.exocad.com. Accessed 4 March 2014

28. Barone, S., Paoli, A., Razionale, A.: Creation of 3D Multi-Body Orthodontic Models by Using Independent Imaging Sensors. Sensors 13(2), 2033-2050 (2013).

29. Sethi, A., Sochor, P.: Predicting esthetics in implant dentistry using multiplanar angulation: a technical note, vol. 10. vol. 4

30. Tarnow, D.P., Eskow, R.N.: Preservation of Implant Esthetics: Soft Tissue and Restorative Considerations. Journal of Esthetic and Restorative Dentistry 8(6), 12-19 (1996). doi:10.1111/j.1708-8240.1996.tb00904.x 
31. Rodriguez-Ciurana, X., Vela-Nebot, X., Segala-Torres, M., Calvo-Guirado, J.L., Cambra, J., MendezBlanco, V., Tarnow, D.P.: The Effect of Interimplant Distance on the Height of the Interimplant Bone Crest When Using Platform-Switched Implants. Int J Periodont Rest 29(2), 141-151 (2009).

32. Teughels, W., Merheb, J., Quirynen, M.: Critical horizontal dimensions of interproximal and buccal bone around implants for optimal aesthetic outcomes: a systematic review. Clin Oral Implan Res 20, 134145 (2009). doi:DOI 10.1111/j.1600-0501.2009.01782.x

33. Traini, T., Novaes, A.B., Piattelli, A., Papalexiou, V., Muglia, V.A.: The relationship between interimplant distances and vascularization of the interimplant bone. Clin Oral Implan Res 21(8), 822-829 (2010). doi:DOI 10.1111/j.1600-0501.2010.01926.x

34. Kourkouta, S., Dedi, K.D., Paquette, D.W., Mol, A.: Interproximal tissue dimensions in relation to adjacent implants in the anterior maxilla: clinical observations and patient aesthetic evaluation. Clin Oral Implan Res 20(12), 1375-1385 (2009). doi:DOI 10.1111/j.1600-0501.2009.01761.x 